\title{
Training data sets for TensorFlow models from TeleEcho data.
}

Dr Bheemaiah, Anil Kumar, A.B Seattle W.A 98125

miyawaki@yopmail.com

\begin{abstract}
:
Data streams are persisted and visualized for a practice of biofeedback based therapy, with the option of @edge decision support for premium services, in the form of on demand tele medical services and CDS based decision support services, and integrated services like Amazon Pharmacy.

Keywords: Digital Medicine, CDS HL7 web hooks, bio-feedback, LSL streams, AWS S3, Wolfram cloud, feature extraction functions, visualization of filters.

What:

Extraction of data by data-mining from hyperscale data from tele-echo data repositories, to create training data sets for specific thread for Tensorflow model templates for transfer learning, with deployment of pre-trained networks using tensorflowlite.

Pre-Trained models are evaluated for prediction accuracy in integrated feature space and classification fitness models, for scalable deployment.
\end{abstract}

How:

We consider the use of TensorFlow Models, and train the models on an EC2 P3 image using GPU computing on SageMaker, using a Thread for the purpose.

We consider the creation of the following :

A MUSE 2 headset for PPG, Gyro Accelerometer data for breath and heart diagnostics is made using a python script and a 1D tensor model.(alexandrebarachant n.d.; "tf.nn.conv1d | TensorFlow Core r2.0" n.d., "tf.keras.layers.Conv1D | TensorFlow Core r2.0" n.d., "Tensorflow - Math behind 1D Convolution with Advanced Examples in TF | Tensorflow Tutorial” n.d.; Lee 2018)

Why:

Digital Medicine is accessible in the mental wellness community with an EEG wearable such as MUSE 2 , which has ppg and accelerometer data which can be data mined with a classifier 1D convolution Tensor Net for detecting any anomalies, requiring telemedicine. 


\section{Introduction.}

Bio-feedback is a wellness technique with more than a century's history in modern medicine and psychiatric practice of wellness. In the context of EEG and ppg/accelerometer and gyro data, the visualization of EEG data and ppg/accelerometer/gyro data, leads to newer versions of biofeedback protocols for wellness by the conscious control of the ppg data, or breathing control reflected in the accelerometer data, by feature extraction to display a feature space as metrics for an easier comprehension of the bio-feedback signals.

Advanced biofeedback, as marketed by MUSE, allows for the conscious control of delta/theta/beta and gamma power density levels, leading to transitions between various bardos of meditation.(Trungpa 1992) In a theoretical formulation of the bardos, late Maharishi Mahesh Yogi,(Maharishi International School n.d.) created a formulation of creative intelligence, inspired by much of the surrealism and abstractionist movements of psychology, with transcendence by meditation, being the home of creativity, the creative intelligence and the states of higher consciousness, defining at least two or maybe more states of transcendence, apart from sleep, awake and dream states or bardos.

Similar definitions exist in Zen and Tibetan

Buddist literature, and unified breath engineering called mindfulness, amenable to bio-feedback practice.(Hanh 2000)(Hanh 2000; McNally 2009)

\section{Problem Definition.}

1. Feature space and visualizations of the features of the 1D waveforms from the ppg and accelerometers and EEG data, both average and differential.

2. Use of a $1 \mathrm{D}$ cnn or r-cnn for categorization of any anomalies, for diagnosis, with predictive analytics for the need for possible early intervention by telemedicine.

\section{Background.}

FeatureExtractorFunction is part of the Feature Extractor command in Wolfram scripting, which allows for the application of a function, either trained or symbolized to create feature space representation of the input waveform. In the case of EEG, ppg and accelerometer data, features are easily represented, in a feature space visualizations with image tags, in wolfram scripting. This helps in biofeedback therapy for wellness apart from visualization of the waveforms.

\section{Conv1D: TensorFlow Network}

Conv1d is a cnn, written in python using keras, to be trained on the training set of data, gathered from teleecho datasets or from other datasets from clinical studies to be conducted in the future. The source code (tensorflow n.d.)

- The Keras functional API

- Train and evaluate with Keras

\section{Muse SDK}

"In addition to EEG, the Muse also provides data from an accelerometer, gyroscope, and, in the case of the Muse 2, a photoplethysmography (PPG) sensor. These data types can be enabled via command line arguments or by passing the 
correct parameters to the stream function. Once enabled, PPG, accelerometer, and gyroscope data will streamed in their own separate LSL streams named "PPG", "ACC", and "GYRO", respectively.

To stream data from all sensors in a Muse 2 from the command line:

muselsl stream --ppg --acc --gyro

As a library function:

from muselsl import stream, list_muses

muses $=$ list_muses ()

stream(muses[0]['address'], ppg_enabled=True,

acc_enabled=True, gyro_enabled=True)

To record data from an alternate data source:

muselsl record --type ACC"

(alexandrebarachant n.d.)

"The lab streaming layer (LSL) is a system for the unified collection of measurement time series in research experiments that handles both the networking, time-synchronization, (near-) real-time access as well as optionally the centralized collection, viewing and disk recording of the data."(sccn n.d.)(“Website” n.d.)

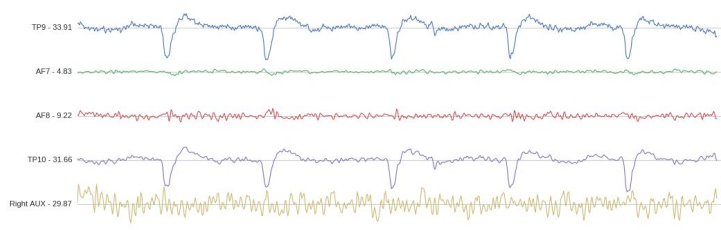

Fig: Pretty printing of stream data for data visualization and use in a Bio-feedback loop.

("Website" n.d.)persisted in the LsL repository and AWS, for analysis using SageMaker, or using the Wolfram cloud.

Muse LSL is an invaluable tool to visualize any LSL data streams from the headset, generalizable to any headset, for bio-feedback.

\section{Discussion.}

Data streams from EEG data and ppg, accelerometer and gyro data are used for breath and pulse diagnosis, creating webhooks for CDS support, along with a practice of bio-feedback by visualization of the stream data.

Feature based visualization of stream data is possible with persistence of the stream data on Wolfram Cloud and analysis with feature extraction functions.

SageMaker based classification for on demand telemedicine is also integrated as a premium feature through VUI interfaces and AWS cloud integration, creating a repository of stream data from teleEcho datasets after the processing to impersonalize the data.("De-Identify Medical Images with the Help of Amazon Comprehend Medical and Amazon Rekognition | Amazon Web Services” 2019) 


\section{Future Work.}

Future work consists of clinical trial of the use of CDS webhooks with a dialog manager for JSON data and a VUI for the use of decision support with datastreams, based on decision support@edge.

\section{References.}

alexandrebarachant. n.d. "Alexandrebarachant/muse-Lsl." GitHub. Accessed October 22, 2019. https://github.com/alexandrebarachant/mus e-lsl.

"De-Identify Medical Images with the Help of Amazon Comprehend Medical and Amazon Rekognition | Amazon Web Services." 2019. Amazon Web Services. March 19, 2019.

https://aws.amazon.com/blogs/machine-lear ning/de-identify-medical-images-with-the-h elp-of-amazon-comprehend-medical-and-a mazon-rekognition/.

Hanh, Thich Nhat. 2000. Plum Village Chanting and Recitation Book.

Lee, Ceshine. 2018. "[Tensorflow] Implementing Temporal Convolutional Networks." Medium. The Artificial Impostor. April 2, 2018.

https://medium.com/the-artificial-impostor/ notes-understanding-tensorflow-part-3-7f66 $33 \mathrm{fcc} 7 \mathrm{c} 7$.

Maharishi International School. n.d. "Science Of Creative Intelligence - Maharishi International School."

Www.maharishischool.ch. Accessed June 4, 2020.

http://www.maharishischool.ch/en/scienceof-creative-intelligence.html\#: :text $=\mathrm{The} \%$
20 founder $\% 20$ of $\% 20$ the $\% 20$ Science, that $\%$ 20 govern $\% 20$ and $\% 20$ maintain $\% 20$ the. McNally, Lama Christie. 2009. The Tibetan Book of Meditation. Harmony.

scen. n.d. "Sccn/labstreaminglayer." GitHub. Accessed June 5, 2020. https://github.com/sccn/labstreaminglayer. tensorflow. n.d. "Tensorflow/tensorflow." GitHub. Accessed June 4, 2020. https:/github.com/tensorflow/tensorflow.

"Tensorflow - Math behind 1D Convolution with Advanced Examples in TF | Tensorflow Tutorial." n.d. Accessed October 22, 2019. https://riptutorial.com/tensorflow/example/ 30750/math-behind-1d-convolution-with-ad vanced-examples-in-tf.

"tf.keras.layers.Conv1D | TensorFlow Core r2.0." n.d. TensorFlow. Accessed October 22, 2019.

https://www.tensorflow.org/api_docs/pytho $\mathrm{n} / \mathrm{tf} /$ keras/layers/Conv1D.

"tf.nn.conv1d | TensorFlow Core r2.0." n.d. TensorFlow. Accessed October 22, 2019. https://www.tensorflow.org/api_docs/pytho $\mathrm{n} / \mathrm{tf} / \mathrm{nn} /$ convld.

Trungpa, Chogyam. 1992. Transcending Madness: The Experience of the Six Bardos. Shambhala Publications.

"Website." n.d. Accessed June 5, 2020. http://doi.org/10.5281/zenodo.3228861. 